\title{
A novel selective medium for the isolation of Burkholderia mallei from equine specimens
}

\author{
Yuta Kinoshita, ${ }^{1,2^{*}}$, Ashley K. Cloutier ${ }^{3}$, David A. Rozak ${ }^{3}$, Md. S. R. Khan², Hidekazu Niwa', Eri Uchida-Fujii ${ }^{1}$, \\ Yoshinari Katayama ${ }^{1}$ and Apichai Tuanyok ${ }^{2}$
}

\begin{abstract}
Background: Burkholderia mallei is a Gram-negative bacterium that causes glanders, a zoonotic disease, especially in equine populations (e.g. horses, donkeys, and mules). B. mallei usually grows slowly on most culture media, and this property makes it difficult to isolate from clinical specimens. One of the problems is that $B$. mallei is easily overgrown by other bacteria, especially in animal specimens collected from non-sterile sites. The aim of this study was to develop a new selective agar for the laboratory diagnosis of glanders. We formulated a new agar, named BM agar, to enrich B. mallei growth, but inhibit the growth of other bacteria and fungi based on their antimicrobial profiles. We compared the growth of B. mallei on BM with Xie's and PC agars, the two previously described selective agars for B. mallei.
\end{abstract}

Results: BM agar could sufficiently grow almost all of the tested B. mallei strains within $72 \mathrm{~h}$ : only one out of the 38 strains grew scantly after $72 \mathrm{~h}$ of incubation. BM agar was further tested with other Burkholderia species and various bacterial species commonly found in the nasal cavities and on the skin of horses. We have found that other Burkholderia species including B. pseudomallei and B. thailandensis can grow on BM agar, but non-Burkholderia species cannot. Furthermore, the specificities of the three selective agars were tested with or without spiking $B$. mallei culture into clinical specimens of non-sterile sites collected from healthy horses. The results showed that BM agar inhibited growths of fungi and other bacterial species better than PC and Xie's agars. We have also found that growth of $B$. mallei on BM agar was equivalent to that on $5 \%$ horse blood agar and was significantly greater than those on the other two agars $(P<0.05)$.

Conclusions: We believe that BM agar can be used to efficiently isolate $B$. mallei from mixed samples such as those typically collected from horses and other contaminated environments.

Keywords: Bacterial isolation, Burkholderia mallei, Equine, Glanders, Horse, Selective medium

\section{Background}

Burkholderia mallei is a Gram-negative bacterium that causes glanders, a zoonotic disease. Glanders is a significant contagious disease, particularly in equine populations (e.g. horses, donkeys, and mules), and other animals (e.g. camels, bears, wolves, and dogs), including humans, are known to be susceptible to $B$. mallei

\footnotetext{
* Correspondence: kinoshita@equinst.go.jp

${ }^{1}$ Microbiology Division, Equine Research Institute, Japan Racing Association,

1400-4 Shiba, Shimotsuke, Tochigi 329-0412, Japan

${ }^{2}$ Department of Infectious Diseases and Immunology, College of Veterinary

Medicine, University of Florida, Gainesville, FL 32608, USA

Full list of author information is available at the end of the article
}

infection [1]. The acute form of glanders is typically observed in donkeys and mules with high fever, respiratory symptoms, and death occurring within a few days. In horses, chronic cases usually develop and horses may endure for several years. Chronic glanders increases the risk of contagion because of the prolonged shedding of B. mallei [1]. International horse transport is becoming more common and, therefore, quarantine testing (e.g. serological test, molecular diagnosis, and bacterial isolation) before and after the transport is very important to prevent the spread of glanders.

(c) The Author(s). 2019 Open Access This article is distributed under the terms of the Creative Commons Attribution 4.0 International License (http://creativecommons.org/licenses/by/4.0/), which permits unrestricted use, distribution, and 
Bacterial isolation is a gold standard method for diagnosing glanders [2]. B. mallei can be grown on most enriched media, including sheep blood agar and trypticase soy agar, but grows very slowly, requiring up to $72 \mathrm{~h}$ of incubation [3]. This property makes it difficult to isolate $B$. mallei from clinical and environmental specimens because $B$. mallei is easily overgrown by other bacteria, especially in animal specimens collected from non-sterile sites $[4,5]$. Therefore, it is recommended to attempt the bacterial isolation from unopend and uncontaminated lesions. However, aseptically collecting specimens is usually difficult because many bacteria and fungi exist as normal flora in respiratory tracts and on skin where the common infections occur. [6, 7]. Additionally, B. mallei is often overgrown by other bacteria even in fresh samples obtained under sterile conditions [8].

A previous report compared four selective media and recommended Pseudomonas cepacia agar (PC agar) for the isolation of $B$. mallei [9]. However, the report also identified important limitations of PC agar, which could lead to difficulties in diagnosing glanders. Four out of 20 B. mallei strains could not grow at all on PC agar and the sizes of 16 colonies, which grew on the agar were quite small $(<1 \mathrm{~mm})$. Similarly, another study has reported that three selective agars, which are basically used for isolation of $B$. pseudomallei, could not grow $20-60 \%$ of the assayed B. mallei strains [10]. Although another semi-selective agar for $B$. mallei was reported by Xie et al. $[3,11]$, the effectiveness of this agar has not been compared with those of other media.

In this study, we developed a new selective medium (Burkholderia mallei agar: BM agar) for laboratory diagnosis of glanders and compared its bacterial selectivity and ability to grow $B$. mallei with previously reported media by testing with bacterial cultures and horses' nasal and skin specimens.

\section{Results}

Bacterial growth on BM agar with pure cultures

Out of the $38 \mathrm{~B}$. mallei strains, 34 strains grew well on BM agar within $48 \mathrm{~h}$, and three strains grew within $72 \mathrm{~h}$. The remaining strain (UCC BURK072) produced colonies after $72 \mathrm{~h}$ incubation, but there were fewer colonies on BM agar than the other strains. Most B. mallei strains formed circular, purple, smooth, and $>1 \mathrm{~mm}$ colonies on BM agar (Fig. 1a and b). All strains of $B$. pseudomallei, B. thailandensis, and Bcc also grew on BM agar within $24 \mathrm{~h}$, whereas growths of all 24 non-Burkholderia species were completely inhibited during the $72 \mathrm{~h}$ incubation period.

\section{Comparison of selective agars with horse samples}

All nasal and skin samples produced numerous commensal bacteria when grown on $5 \%$ horse blood agar. Furthermore, these samples produced at least one bacterial colony on Xie's agar, while 7 and 3 nasal swabs grew bacteria on $\mathrm{PC}$ and $\mathrm{BM}$ agars, respectively (Table 1). None of the skin swabs grew bacteria on $\mathrm{PC}$ or $\mathrm{BM}$ agar. Growths of fungi were found from 12 nasal and 7 skin swabs on Xie's agar, and from 1 nasal and 2 skin swabs on PC agar. No fungi were isolated from any of the tested nasal and skin swabs on BM agar. The following bacterial species were identified on the different agars: Xie's agar, Chryseobacterium gleum, Bacillus cereus, Arthrobacter histidinolovorans, Paenibacillus species, Bacillus species, Enterobacter cloacae, Rhizobium radiobacter, Staphylococcus saprophyticus, Enterococcus casseliflavus, Pantoea ananatis, Pantoea agglomerans, Staphylococcus species, Staphylococcus haemolyticus, Sphingomonas species, Rhizobium species, and Escherichia coli; PC agar,

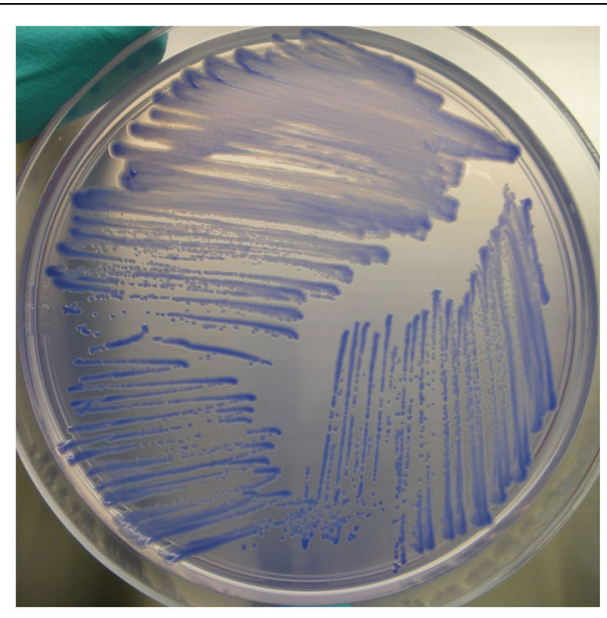

a

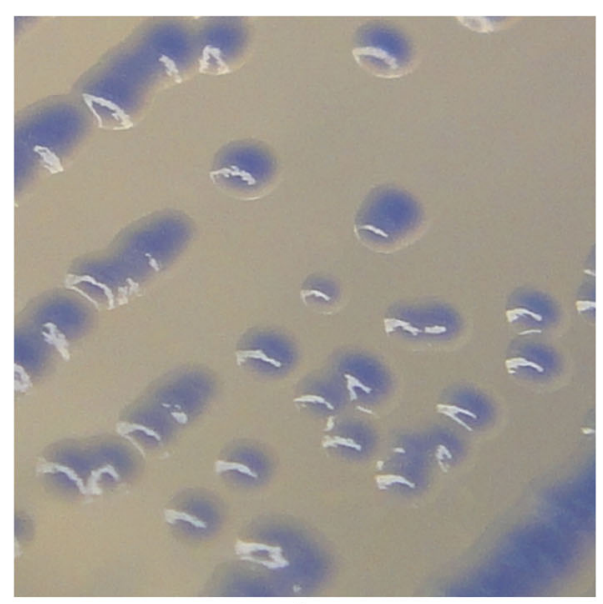

b

Fig. 1 Representative growth of B. mallei GTC 3P0003 (a) plate view and (b) colony view, on BM agar after $72 \mathrm{~h}$ incubation at $37^{\circ} \mathrm{C}$ 
Table 1 Numbers of agars on which at least one colony of bacteria or fungi grew

\begin{tabular}{llllll}
\hline Medium & \multicolumn{2}{l}{ Nasal swab $(n=34)$} & & \multicolumn{2}{l}{ Skin swab $(n=34)$} \\
\cline { 2 - 3 } \cline { 6 - 7 } & Bacteria & Fungi & & Bacteria & Fungi \\
\hline Xie's agar & $34(100 \%)$ & $12(35.3 \%)$ & & $34(100 \%)$ & $7(20.6 \%)$ \\
PC agar & $7(20.6 \%)^{a}$ & $1(2.9 \%)^{a}$ & & $0(0 \%)^{*}$ & $2(5.9 \%)$ \\
BM agar & $3(8.8 \%)^{a}$ & $0(0 \%)^{a}$ & & $0(0 \%)^{*}$ & $0(0 \%)^{*}$ \\
\hline
\end{tabular}

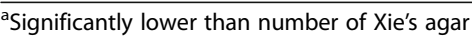

Klebsiella pneumoniae, Pseudomonas putida, Pseudomonas koreensis, and Pantoea agglomerans; BM agar, Herbaspirillum huttiense.

\section{Comparison of growth efficacy}

To assess how well BM agar can support B. mallei growth we tested three $B$. mallei strains (GTC $3 \mathrm{P} 0003^{\mathrm{T}}$, GTC 3P0016, and GTC 3P0078) for growth on all the three selective agars and blood agar at 37 ${ }^{\circ} \mathrm{C}$ for $72 \mathrm{~h}$. Plating serial dilutions of the same three $B$. mallei cultures on each of the agars, we computed the average bacterial concentration of the original cultures to be $1.48 \times 10^{8}, 8.03 \times 10^{7}, 8.03 \times 10^{6}$ and $1.38 \times 10^{8} \mathrm{CFU} / \mathrm{mL}$ when using blood, Xie's, PC, and $\mathrm{BM}$ agars, respectively (Fig. 2). We noted that the average colony number of three $B$. mallei strains on BM agar was equivalent to that on $5 \%$ horse blood agar and was significantly greater than those on the other two agars $(p$-value $<0.05)$.

\section{Comparison of culture appearances}

Appearances of the four agars by using spiked or non-spiked specimens are shown in Fig. 3. The un-spiked nasal swab did not grow any bacteria or fungi on both PC and BM agars, while several kinds of bacteria or fungi were found on blood agar and Xie's agar. In the spiked specimen, these commensal bacteria and fungi on Xie's agar covered B. mallei colonies, making it difficult to pick up B. mallei colonies. Concerning $\mathrm{PC}$ and $\mathrm{BM}$ agar, one colony of $B$. mallei was found on PC agar, whereas more than 10 colonies of $B$. mallei were observed on BM agar. The colonies were confirmed as $B$. mallei by the MALDI Biotyper System.

\section{Discussion}

Although glanders has been eradicated from many countries over the last century, several outbreaks of glanders still occurred in the equine population in Asia, Middle-East, Africa, and South America [1, 12]. The international movement of horses increases the risks of spreading exotic diseases, and many cases of disease transfer, including glanders, have been identified [13, 14]. Therefore, quarantines before and after the international transport are quite important. Concerning equine glanders, combination of complement fixation test and western blot is usually performed to check the serological status of horses [3], but a gold standard method for diagnosing this disease remains the isolation of the causative bacterium, B. mallei from clinical specimens [2]. One of the important problems of $B$. mallei is its difficulty to isolate the bacteria from environmental samples. Investigators are often unable to isolate $B$. mallei from seropositive or species-specific PCR positive horses $[15,16]$. The difficulty of isolating B. mallei is mainly attributed to its slow growing character [3]. The

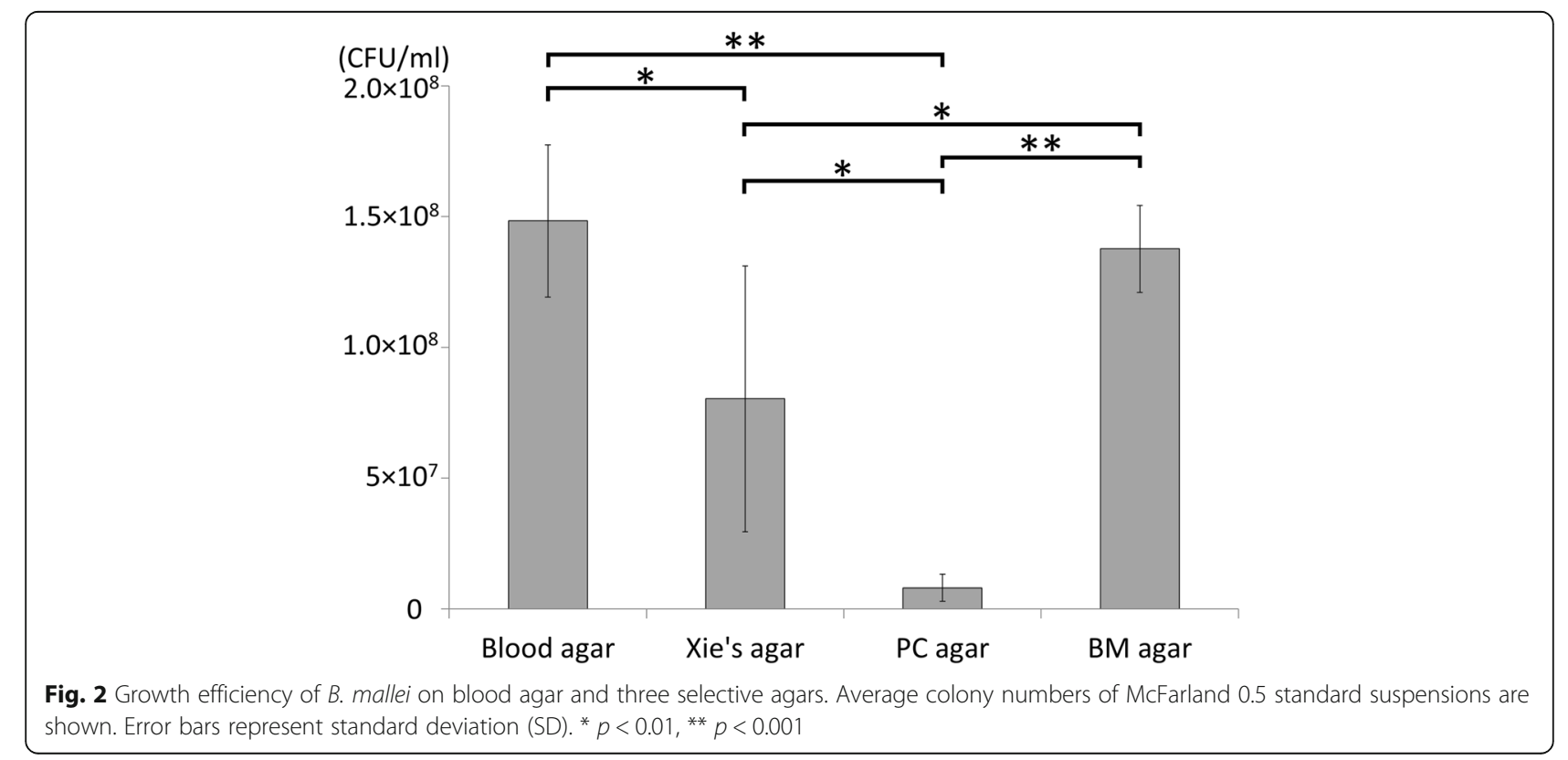




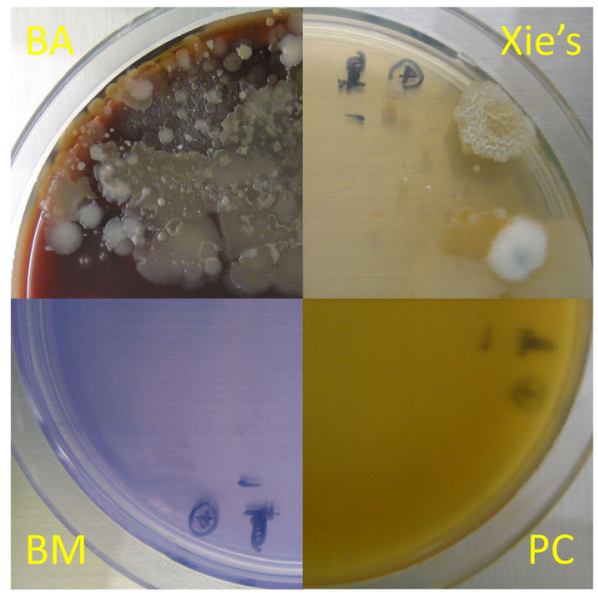

a

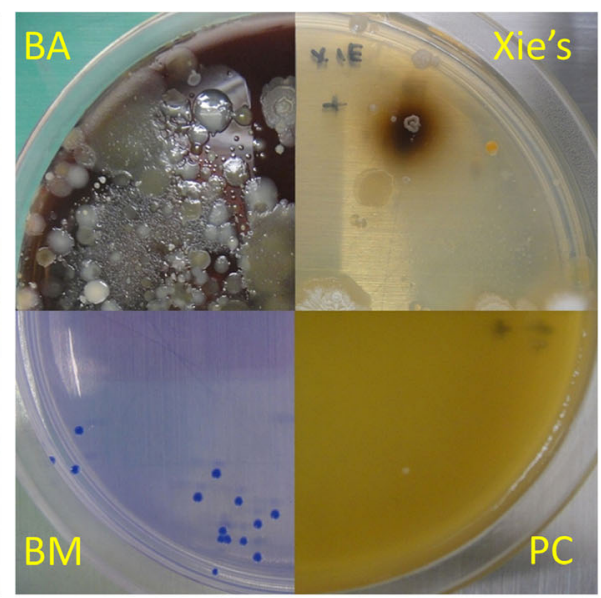

b

Fig. 3 Comparison of culture appearances by using horse nasal swab, (a) un-spiked specimen, and (b) specimen spiked with B. mallei GTC 3P0003 ${ }^{\top}$. This figure shows that blood agar and Xie's agar grew other bacteria or fungi, making it difficult to pick up B. mallei colonies. BM agar and $P C$ agar can efficiently inhibit growths of non-B. mallei isolates. BM agar has the benefit of supporting higher colony numbers, compared with PC agar

bacteria's growth limitations on commonly used selective media are also considered a reason for the challenges associated with isolating B. mallei. For example, B. pseudomallei, a genetically closely related species to $B$. mallei, is often isolated on Ashdown's agar, which uses crystal violet and gentamicin as selective agents $[17,18]$. However, B. mallei growth generally fails on Ashdown's agar [9] because the minimum inhibitory concentration of gentamicin for $B$. mallei is significantly lower than that for B. pseudomallei $[19,20]$. Similarly, MacConkey agar, which is commonly used to isolate Gram-negative and enteric bacilli, is generally not useful for isolating $B$. mallei because the bacteria may or may not grow on this agar [21]. The PC and Xie's agars are useful in isolating B. mallei from contaminated clinical samples [3, 9], but these agars have their own limitations. PC agar could not grow some strains of $B$. mallei and its colonies formed on this agar were very small $(<1 \mathrm{~mm})$ even after $72 \mathrm{~h}$ incubation. In addition, the effectiveness of Xie's agar has not been compared with those of other agars. Therefore, we have developed a new selective agar "BM" to isolate $B$. mallei from equine specimens and compared its selectivity and growth efficiency with the previously recommended selective agars. Although non-selective agars like glycerol dextrose agar and glycerol potato agar can be used to grow B. mallei, we excluded these from our study, which focused on using selective agars to isolate B. mallei from heavily contaminated samples.

Concerning the formulation of BM agar, glycerol is well known to enhance the growth of $B$. mallei [3], and crystal violet and cycloheximide are used to inhibit some Gram-positive bacteria and fungi. In particular, a combination of antimicrobials is quite important to make a medium with high selectivity. B. mallei was reportedly resistant to all the three antimicrobials (ticarcillin, fosfomycin, and polymyxin B) [20, 22]. Ticarcillin has antibacterial activity against Gram-positive bacteria, while polymyxin B acts against Gram-negative bacteria, and fosfomycin affects both Gram-positive and Gram-negative bacteria. Although $B$. mallei was reported to be intrinsically resistant to polymyxin B [22], some B. mallei strains showed its relative susceptibility to this antimicrobial in our preliminary test. Specifically, polymyxin B of greater than 70,000 units/L could slow down the growths of $B$. mallei strains (data not shown). Therefore, the concentration of polymyxin B which did not affect the bacterial growths was used in this study. In addition, this view could make an impact on the difference of growth efficacy between BM agar and PC agar as described below, because PC agar contains high concentration of polymyxin B $(300,000$ units/L).

Our results with bacterial pure cultures showed that the BM agar could allow 34 out of $38 \mathrm{~B}$. mallei strains to grow within $48 \mathrm{~h}$. Three of the other four strains needed up to $72 \mathrm{~h}$ to be easily picked up from the agar plate. The remaining one grew scantly even after $72 \mathrm{~h}$, indicating that at least $72 \mathrm{~h}$ incubation is recommended for isolation of $B$. mallei by using BM agar. Recovery of the slow-growing strain might benefit from pre-enriching the clinical sample or increasing the incubation time. Other Burkholderia species tested were also grown on BM agar. Although Burkholderia species are not frequently isolated from equine specimens $[6,23,24]$, skin samples especially might contain Burkholderia species because most species of genus Burkholderia naturally exist in soil [25, 26]. Therefore, additional confirmations, such as specific PCR 
test, are needed after isolating B. mallei-suspected colonies from BM agar. Concerning non-Burkholderia species, BM agar showed its high specificity by inhibiting the growths of common bacteria frequently found from equine specimens. The results of comparison tests using equine nasal and skin swabs also illustrate the superiority of BM agar. BM agar can inhibit the growth of both bacteria and fungi more efficiently than PC and Xie's agar. Several Gram-negative bacteria grew on PC agar while both Gram-positive and Gram-negative bacteria grew on Xie's agar. Although some non-Burkholderia species, such as Herbaspirillum huttiense, were found on BM agar, its colony number was relatively low and did not complicate the isolation of $B$. mallei from BM agar.

Regarding growth efficiency of $B$. mallei, high effectiveness is also needed for isolating this pathogen because the concentration of $B$. mallei in clinical specimens of the infected equid is low $[1,14]$. Our result revealed that BM agar was as effective as the $5 \%$ horse blood agar. Comparing to the $\mathrm{PC}$ and Xie's agar, a significantly greater number of $B$. mallei colonies can be grown on $\mathrm{BM}$ agar. In addition to BM agar's specificity, the agar's high recovery rate makes it useful for isolating $B$. malle $i$ from horse specimens. Therefore, focusing on combined approaches for the detection of B. mallei and B. pseudomallei, BM agar could be used as a unique selective medium that can efficiently grow these two pathogenic bacteria and inhibit the growth of non-Burkholderia species.

\section{Conclusions}

Our study has shown that BM agar is a novel selective agar for effectively isolating $B$. mallei from horse specimens. We believe that this new agar can be used to provide sensitive and selective isolation of $B$. mallei for both diagnosis and future research.

\section{Methods}

\section{Culture media}

$\mathrm{BM}$ agar was prepared by combining $5 \mathrm{~g}$ of proteose peptone no.3, $10 \mathrm{~g}$ of tryptone pancreatic digest of casein, $0.25 \mathrm{~g}$ of magnesium sulfate, $1 \mathrm{~g}$ of sodium pyruvate, $5 \mathrm{~g}$ of sodium chloride, $40 \mathrm{~mL}$ of glycerol, $3 \mathrm{mg}$ of crystal violet ( $3 \mathrm{~mL}$ from $0.1 \% \mathrm{w} / \mathrm{v}$ crystal violet in distilled water), 50 $\mathrm{mg}$ of cycloheximide ( $5 \mathrm{~mL}$ from $1 \% \mathrm{w} / \mathrm{v}$ cycloheximide in absolute ethanol), $15 \mathrm{~g}$ of agar, and distilled water (960 $\mathrm{ml})$. The mixture was sterilized at $121^{\circ} \mathrm{C}$ for $15 \mathrm{~min}$. After cooling to $45^{\circ} \mathrm{C}$, the medium was supplemented with three antimicrobials: $16.7 \mathrm{mg}$ of ticarcillin disodium salt ( $668 \mu \mathrm{L}$ from $25 \mathrm{mg} / \mathrm{ml}$ stock solution), $197.8 \mathrm{mg}$ of fosfomycin sodium $(1520 \mu \mathrm{L}$ from $130 \mathrm{mg} / \mathrm{mL}$ stock solution), 40,000 units of polymyxin B $(400 \mu \mathrm{L}$ from 100,000 units/ $\mathrm{mL}$ stock solution). The formulations of $\mathrm{PC}$ agar and Xie's agar were based on previous reports $[9,11]$.
Additional file 1 :Table S1 compares the formulations of the BM, PC, and Xie's agars used in this study.

\section{Bacteria used in this study}

A collection of 68 isolates of Burkholderia species and 24 isolates of non-Burkholderia species was used for evaluation of BM agar (see Additional file 2 :Table S2). These Burkholderia species included 38 B. mallei, 5 B. pseudomallei, 6 B. thailandensis, and 19 strains of Burkholderia cepacia complex (Bcc). The non-Burkholderia species, which are frequently isolated from horse specimens, were tested in this study and the collection was composed of three strains from each of the following eight bacterial species (total 24 strains): Pseudomonas aeruginosa, Stenotrophomonas maltophilia, Escherichia coli, Klebsiella pseumoniae, Streptococcus zooepidemicus, Streptococcus equi, methicillin-susceptible Staphylococcus aureus, and methicillin-resistant Staphylococcus aureus.

\section{Bacterial growth on BM agar with pure cultures}

Prior to testing, each of the strains were subcultured onto blood agar or LB agar containing 4\% glycerol. For Burkholderia species, one colony of each strain was picked up and subcultured onto BM agar. The plates were incubated at $37^{\circ} \mathrm{C}$ for $72 \mathrm{~h}$. For non-Burkholderia species, each isolate was suspended in $1 \mathrm{~mL}$ of saline solution $(0.85 \% \mathrm{w} / \mathrm{v})$ to a turbidity equivalent to a McFarland 0.5 standard (approximately $1.5 \times 10^{8} \mathrm{CFU} / \mathrm{mL}$ ). Each suspension was diluted 1000-fold (approximately $1.5 \times 10^{5} \mathrm{CFU} / \mathrm{mL}$ ) and a $100 \mu \mathrm{L}$ aliquot of the dilution (approximately $1.5 \times 10^{4} \mathrm{CFU} / 100 \mu \mathrm{L}$ ) was inoculated onto the medium and spread with a plate spreader. Then, the plates were incubated at $37^{\circ} \mathrm{C}$ for $72 \mathrm{~h}$. The numbers of bacterial colonies were counted every $24 \mathrm{~h}$.

\section{Collection and analysis of horse samples}

A total of 34 nasal swabs and 34 skin swabs were sampled from 17 healthy horses that were kept by Equine research institute; both sides of the nasal cavity and shoulder skin were sampled from each horse. Each swab was dipped into $500 \mu \mathrm{L}$ of distilled water, and $100 \mu \mathrm{L}$ of the suspension was inoculated onto the three selective agars (BM, PC, and Xie's) and 5\% horse blood agar. Growths of bacteria and fungi were checked after incubating at $37^{\circ} \mathrm{C}$ for $72 \mathrm{~h}$. Bacterial isolates from horse specimens were identified by matrix assisted laser desorption ionization-time of flight mass spectrometry with MALDI Biotyper System (Bruker Daltonics, Leipzig, Germany), in accordance with the manufacturer's instructions. The Biotyper software 3.1 and the MALDI Biotyper reference library version 5.0.0.0. were used in this study. The bacterial identifications were interpreted according to the criteria of the manufacturer, namely 
species level, identification score of $\geq 2.000$; genus level, $\geq 1.700$ to $<2.000$; no identification, $<1.700$.

\section{Evaluation of growth efficiencies}

Three B. mallei strains (GTC 3P0003 ${ }^{\mathrm{T}}$, GTC 3P0016, and GTC 3P0078) obtained from Gifu Type Culture Collection were used to compare growth efficiencies of $B$. mallei in this study. Each strain was suspended in saline solution $(0.85 \% \mathrm{w} / \mathrm{v})$ to a turbidity equivalent to a McFarland 0.5 standard (original suspension). Ten-fold serial dilutions of the suspensions were inoculated in triplicate onto four agars (5\% horse blood agar, BM agar, PC agar, and Xie's agar) and the average colony counts of the original suspensions were separately calculated for each of the agars after incubation at $37^{\circ} \mathrm{C}$ for $72 \mathrm{~h}$.

\section{Comparison of culture appearances}

To compare the appearances of bacteria grown on each of the four agars, B. mallei GTC $3 \mathrm{P}_{0003}{ }^{\mathrm{T}}$ was suspended in $1 \mathrm{~mL}$ of phosphate-buffered saline (PBS) to a turbidity equivalent to a McFarland 0.5. Ten-fold serial dilutions of the suspension were prepared. One nasal swab obtained from a healthy horse was dipped into $2.5 \mathrm{~mL}$ of PBS. Then, $10 \mu \mathrm{L}$ of each bacterial dilution and $200 \mu \mathrm{L}$ of the swab suspension were mixed and $50 \mu \mathrm{L}$ aliquots of the mixture were inoculated onto the four agars. Appearances of the agars were observed after incubation at $37^{\circ} \mathrm{C}$ for $72 \mathrm{~h}$. The same amount of nasal swab suspension was also inoculated onto the four agars without being spiked with $B$. mallei, and bacterial and fungal colonies were checked after incubation for comparison.

\section{Statistics}

A Fisher's exact test and t-test were conducted by using Excel Statistics ver. 7.0 (Esumi Co., Ltd., Tokyo, Japan) for statistical analysis of comparisons. A $p$-value of $<$ 0.05 was considered to indicate a significant difference.

\section{Additional files}

Additional file 1: Table S1. Constituents of three selective agars.

(XLSX $12 \mathrm{~kb}$ )

Additional file 2: Table S2. Bacteria used in this study. (XLSX $14 \mathrm{~kb}$ )

\section{Abbreviations}

Bcc: Burkholderia cepacia complex; BM agar: Burkholderia mallei agar; GTC: Gifu Type Culture Collection; PC agar: Pseudomonas cepacia agar; UCC: The United States Army Medical Research Institute for Infectious Diseases' Unified Culture Collection

\section{Acknowledgements}

We thank Dr. Jirarat Songsri of Walailak University in Thailand for providing various BCC strains for testing in this study. Opinions, interpretations, conclusions, and recommendations are those of the authors and are not necessarily endorsed by the United States Army.

\section{Funding}

This study was supported by the Japan Racing Association. The funding body provided all the help in the design of the study and collection, analysis, and interpretation of data and in writing the manuscript.

\section{Availability of data and materials}

The datasets used and/or analyzed during the current study are available from the corresponding author on reasonable request.

\section{Authors' contributions}

YK performed and analyzed the experiments and drafted the manuscript. AKC, DAR, and MSRK performed the laboratory test and helped to revise the manuscript. HN, EUF, and YK helped to draft and revise the manuscript. AT participated in the data analysis and results interpretation and helped to draft and revise the manuscript. All authors critically read and approved the final version.

\section{Ethics approval}

All experiments were conducted in accordance with ethical and welfare regulations of the Animal care committee of the Equine Research Institute and ethics regarding this study were approved by the committee under the identification number 19-19.

Consent for publication

Not applicable

\section{Competing interests}

The authors declare that they have no competing interest.

\section{Publisher's Note}

Springer Nature remains neutral with regard to jurisdictional claims in published maps and institutional affiliations.

\section{Author details}

Microbiology Division, Equine Research Institute, Japan Racing Association, 1400-4 Shiba, Shimotsuke, Tochigi 329-0412, Japan. ²Department of Infectious Diseases and Immunology, College of Veterinary Medicine, University of Florida, Gainesville, FL 32608, USA. ${ }^{3}$ Unified Culture Collection, Diagnostic Systems Division, U.S. Army Medical Research Institute of Infectious Diseases, Fort Detrick, Frederick, MD 21702-5011, USA.

Received: 21 December 2018 Accepted: 18 April 2019

Published online: 07 May 2019

\section{References}

1. Khan I, Wieler LH, Melzer F, Elschner MC, Muhammad G, Ali S, Sprague LD, Neubauer H, Saqib M. Glanders in animals: a review on epidemiology, clinical presentation, diagnosis and countermeasures. Transbound Emerg Dis. 2013;60(3):204-21.

2. Blue SR, Pombo DJ, Woods ML: Glanders and melioidosis. In: Zoonoses: biology, clinical practice and public health control. 2nd edn. Edited by Palmer SR, Soulsby L, Simpson DIH. Oxford, UK: Oxford University Press; 1998: 105-113.

3. OIE Terrestrial Manual, Glanders. Chapter 3.5.11 [http://www.oie.int/en/ international-standard-setting/terrestrial-manual/access-online/].

4. Wernery U, Wernery R, Joseph M, Al-Salloom F, Johnson B, Kinne J, Jose S, Tappendorf B, Hornstra H, Scholz HC. Natural Burkholderia mallei infection in dromedary, Bahrain. Emerg Infect Dis. 2011;17(7):1277-9.

5. Verma AK, Saminathan M, Neha TR, Dhama K, Singh SV. Glanders-a reemerging zoonotic disease: a review. J Biol Sci. 2014;14(1):38-51.

6. Adams MK, Hendrickson DA, Rao S, Popelka FO, Bolte D. The Bacteria isolated from the skin of 20 horses at a veterinary teaching hospital. J Equine Vet Sci. 2010;30(12):687-95.

7. Hoquet F, Higgins R, Lessard P, Vrins A, Marcoux M. Comparison of the bacterial and fungal flora in the pharynx of normal horses and horses affected with pharyngitis. Can Vet J. 1985;26(11):342-6.

8. Wernery U: Glanders. In: Infectious Diseases of the Horse. edn. Edited by S. MT, E. HR. Cambridgeshire, UK: Equine Veterinary Journal Ltd; 2009: 253-260.

9. Glass MB, Beesley CA, Wilkins PP, Hoffmaster AR. Comparison of four selective media for the isolation of Burkholderia mallei and Burkholderia pseudomallei. Am J Trop Med Hyg. 2009;80(6):1023-8. 
10. Edler C, Derschum H, Kohler M, Neubauer H, Frickmann H, Hagen RM. Comparison of mast Burkholderia Cepacia, Ashdown + gentamicin, and Burkholderia Pseudomallei Selective agar for the Selective growth of Burkholderia Spp. Eur J Microbiol Immunol (Bp). 2017;7(1):15-36.

11. Xie X, Xu F, Xu B, Duan X, Gong R: A New Selective Medium for Isolation of Glanders Bacilli. Collected papers of veterinary research. Control Institute of Veterinary Biologics, Ministry of Agriculture, Peking, China (People's Rep. of). 1980(6):83-90.

12. Elschner MC, Neubauer H, Sprague LD. The resurrection of Glanders in a new epidemiological scenario: a beneficiary of "global change". Curr Clin Microbiol Rep. 2017:4(1):54-60.

13. Dominguez M, Munstermann S, de Guindos I, Timoney P. Equine disease events resulting from international horse movements: systematic review and lessons learned. Equine Vet J. 2016;48(5):641-53.

14. Kettle AN, Wernery U. Glanders and the risk for its introduction through the international movement of horses. Equine Vet J. 2016;48(5):654-8.

15. Malik P, Singha H, Goyal S, Khurana SK, Tripathi BN, Dutt A, Singh D, Sharma $\mathrm{N}$, Jain S: Incidence of Burkholderia mallei infection among indigenous equines in India. Vet Rec Open. 2015; 24(2(2)):e000129.

16. Elschner M, Klaus CU, Liebler-Tenorio E, Schmoock G, Wohlsein P, Tinschmann O, Lange E, Kaden V, Klopfleisch R, Melzer F, et al. Burkholderia mallei infection in a horse imported from Brazil. Equine Vet Educ. 2009;21(3):147-50

17. Walsh AL, Wuthiekanun V. The laboratory diagnosis of melioidosis. Br J Biomed Sci. 1996;53(4):249-53.

18. Lau SK, Sridhar S, Ho CC, Chow WN, Lee KC, Lam CW, Yuen KY, Woo PC Laboratory diagnosis of melioidosis: past, present and future. Exp Biol Med (Maywood). 2015;240(6):742-51.

19. Kenny DJ, Russell P, Rogers D, Eley SM, Titball RW. In vitro susceptibilities of Burkholderia mallei in comparison to those of other pathogenic Burkholderia spp. Antimicrob Agents Chemother. 1999;43(11):2773-5.

20. Thibault FM, Hernandez E, Vidal DR, Girardet M, Cavallo JD. Antibiotic susceptibility of 65 isolates of Burkholderia pseudomallei and Burkholderia mallei to 35 antimicrobial agents. J Antimicrob Chemother. 2004;54(6):1134-8.

21. Gilad J, Schwartz D, Amsalem Y. Clinical features and laboratory diagnosis of infection with the potential bioterrorism agents Burkholderia mallei and Burkholderia pseudomallei. Int J Biomed Sci. 2007:3(3):144-52.

22. Schweizer HP, Peacock SJ. Antimicrobial drug-selection markers for Burkholderia pseudomallei and B. mallei. Emerg Infect Dis. 2008;14(11):1689-92.

23. Hoffman AM, Viel L, Prescott JF, Rosendal S, Thorsen J. Association of microbiologic flora with clinical, endoscopic, and pulmonary cytologic findings in foals with distal respiratory tract infection. Am J Vet Res. 1993; 54(10):1615-22.

24. Boguta L, Gradzki Z, Borges E, Maurin F, Kodjo A, Winiarczyk S. Bacterial flora in foals with upper respiratory tract infections in Poland. J Vet Med B Infect Dis Vet Public Health. 2002;49(6):294-7.

25. Coenye T, Vandamme P. Diversity and significance of Burkholderia species occupying diverse ecological niches. Environ Microbiol. 2003;5(9):719-29.

26. Eberl L, Vandamme P. Members of the genus Burkholderia: good and bad guys. F1000Res. 2016;5:1007.

Ready to submit your research? Choose BMC and benefit from:

- fast, convenient online submission

- thorough peer review by experienced researchers in your field

- rapid publication on acceptance

- support for research data, including large and complex data types

- gold Open Access which fosters wider collaboration and increased citations

- maximum visibility for your research: over $100 \mathrm{M}$ website views per year

At BMC, research is always in progress.

Learn more biomedcentral.com/submissions 